Canadian Science Publishing

Applied Physiology, Nutrition, and Metabolism Physiologie appliquée, nutrition et métabolisme

\title{
Do elite breath-hold divers suffer from mild short-term memory impairments?
}

\begin{tabular}{|c|c|}
\hline Journal: & Applied Physiology, Nutrition, and Metabolism \\
\hline Manuscript ID & apnm-2017-0245.R2 \\
\hline Manuscript Type: & Article \\
\hline Date Submitted by the Author: & 18-Sep-2017 \\
\hline Complete List of Authors: & $\begin{array}{l}\text { Billaut, François; Université Laval, Département de kinésiologie } \\
\text { Gueit, Patrice; Universite de Rouen, Centre d'Etudes des Transformations } \\
\text { des Activités Physiques et Sportives, EA n³832 } \\
\text { Faure, Sylvane; Universite de Nice Sophia Antipolis, Laboratoire } \\
\text { d'Anthropologie et de Psychologie Cognitives et Sociales, EA } 7278 \\
\text { Costalat, Guillaume; Universite de Picardie Jules Verne, Laboratoire } \\
\text { Adaptations Physiologiques à I'Exercice et Réadaptation à I'effort (APERE), } \\
\text { EA n³300 } \\
\text { Lemaitre, Frédéric; Universite de Rouen UFR des Sciences et Techniques, } \\
\text { Centre d'Etudes des Transformations des Activités Physiques et Sportives, } \\
\text { EA n³832 }\end{array}$ \\
\hline \multicolumn{2}{|l|}{$\begin{array}{r}\text { Is the invited manuscript for } \\
\text { consideration in a Special } \\
\text { Issue? : }\end{array}$} \\
\hline Keyword: & breath-hold diving, apnea, cognition, short-term memory, hypoxia \\
\hline
\end{tabular}


Title: Do elite breath-hold divers suffer from mild short-term memory impairments?

Authors: François Billaut ${ }^{1}$, Patrice Gueit ${ }^{2}$, Sylvane Faure ${ }^{3}$, Guillaume Costalat ${ }^{4}$, Frédéric Lemaître $^{2}$

\section{Authors affiliations:}

${ }^{1}$ Département de kinésiologie, Faculté de Médecine, Université Laval, Québec, QC, Canada

${ }^{2}$ Centre d'Etudes des Transformations des Activités Physiques et Sportives, EA n³832, Faculté des Sciences du Sport et de l'Education Physique de Rouen, Université de Rouen, France $\square$

${ }^{3}$ Laboratoire d'Anthropologie et de Psychologie Cognitives et Sociales, EA 7278, Université Nice Sophia-Antipolis.

${ }^{4}$ Laboratoire Adaptations Physiologiques à l'Exercice et Réadaptation à l'effort (APERE), EA n³300, Université de Picardie Jules Verne, France

\section{Address for correspondence:}

A/Prof François Billaut

Département de kinésiologie, Université Laval

2300, rue de la Terrasse, Québec, QC, G1V 0A6, Canada

Email: francois.billaut@kin.ulaval.ca 


\begin{abstract}
Repeated apneas are associated with severe hypoxemia that may ultimately lead to loss of consciousness in some breath-hold divers. Despite increasing number of practitioners, the relationship between apnea-induced hypoxia and neurocognitive functions is still poorly understood in the sport of free diving. To shed light onto this phenomenon, we examined the impact of longterm breath-hold diving training on attentional processing, short-term memory, and long-term mnesic and executive functions. Thirty-six men matched for age, height and weight were separated into three groups [12 elite breath-hold divers (EBHD, mean static apnea best time 371 sec, 105 months mean apnea experience); 12 novice breath-hold divers (NBHD, mean best time 243 sec, 8.75 months mean apnea experience); and 12 physical education students with no breath-hold diving experience (CRTL)], and performed varied written and computerized neuropsychological tasks. Compared with the two other groups, EBHD were slower to complete the interference card during a Stroop test $(\mathrm{F}(1,33)=4.70, \mathrm{p}<0.05)$, and presented more errors on the interference card $(\mathrm{F}(1,33)=2.96, \mathrm{p}<0.05)$ and a lower total interference score $(\mathrm{F}(1,33)=5.64, \mathrm{p}<0.05)$. The time to complete the interference card test was positively correlated with maximal static apnea duration $(\mathrm{r}=0.73, \mathrm{p}<0.05)$ and the number of years of breath-hold diving training $(\mathrm{r}=0.79, \mathrm{p}<0.001)$. These findings suggest that breath-hold diving training over several years may cause mild, but persistent, short-term memory impairments.
\end{abstract}

Key words: breath-hold diving, apnea, hypoxia, cognition, short-term memory 


\section{Introduction}

During static apnea, breath-hold (BH) divers hold their breath for as long as possible with the mouth and nose submerged throughout the duration of the apnea (current male World record: $11 \mathrm{~min} 35 \mathrm{sec}$, established in 2009). The major hazard in BH diving is to lose consciousness from hypoxia if the $\mathrm{BH}$ episode is too long. Negative neurological events subsequent to severe brain hypoxia include loss of motor control and consciousness with a prevalence during static $\mathrm{BH}$ competition of $9.6 \%$ and $1.1 \%$, respectively. (Lindholm 2007). Both animal and human studies have implicated hypoxia as a critical factor in neuronal dysfunction (Caine and Watson 2000, Hornbein 2001). Surprisingly however, despite the increasing number of practitioners in the sports of free diving, the potentially negative impact of a reduction in the supply of $\mathrm{O}_{2}$ to the brain on short- and long-term brain functioning has not received much attention.

There is compelling evidence demonstrating the deleterious effects of acute and chronic exposure to hypoxia on a range of cognitive and behavioural performances (Caine and Watson 2000, Auer and Sutherland 2002). For examples, neuropsychological testing revealed a decline in both visual and verbal long-term memory after mountain ascent (Hornbein et al. 1989) as well as in the perceptual ability of pilots under hypoxic conditions corresponding to $5000 \mathrm{~m}$ altitude (Truszczyński et al. 2009). To our best knowledge, only one study has explored the relationship between BH diving training and cognitive performances. Ridgway and colleagues (Ridgway and McFarland 2006) did not find any pathological scores in 12 male and 9 female BH divers (mean static apnea best time 294s) during an exhaustive neuropsychological evaluation including speed of visuo-motor responding, speed of language, comprehension, response inhibition, visual and verbal attention, working memory and episodic memory. This evaluative study also failed to demonstrate any correlation between the number of negative neurological events and cognitive results. In addition, no difference was detected in this evaluation between $\mathrm{BH}$ divers practising apnea for less than 4 years vs. 4-20 years, suggesting that 1-20 years of repeated exposures to hypoxia does not impact 
cognition as measured via standard neuropsychological tasks. However, although this neuropsychological investigation may not have detected cognitive alterations, a brain imaging study showed abnormal single photon emission computed tomographic scans suggesting brain function abnormalities in 5 elite BH divers (Potkin and Uszler 2006). This result is in good agreement with the increase in $\mathrm{S} 100$ calcium binding protein $\beta$ concentration (S100B, a serum marker for cerebral ischemia and brain damage) measured after maximal $\mathrm{BH}$ episodes in trained $\mathrm{BH}$ divers who were able to sustain significant arterial hypoxemia (Andersson et al. 2009). The large difference in training status and performance capabilities between divers, as well as the number of recently incurred negative neurological events may account for these apparent discrepancies. Nonetheless, there appears to be a clear likelihood for, at least mild, neurocognitive sequelae in elite $\mathrm{BH}$ divers that warrants further investigation.

Therefore, the aim of the present study was to examine the potentially deleterious impact of repeated maximal $\mathrm{BH}$ episodes induced by apnea training on neurocognitive performances. We hypothesized that elite $\mathrm{BH}$ divers would score lower in neuropsychological tasks compared to novice $\mathrm{BH}$ divers and non-diving controls.

\section{Material and methods}

\section{Participants}

All experimental procedures in this study were approved by the local research ethics committee. The participants were informed of the objectives and procedures of the study, and all gave written consent prior to the start of the experiment. Thirty-six healthy, non-smoker men were recruited from the French apnea diving association and the University of Nice (all of them were French-native speakers). Participants were separated into 3 groups: 12 elite breath-hold divers (EBHD); 12 novice breath-hold divers (NBHD); and 12 participants without any experience in breath-hold diving (CRTL). All anthropometric parameters and experience statistics are presented in table 1. The 
EBHDs were able to perform a static and dynamic apnea longer than 6 minutes and 100 meters, respectively, and practiced apnea for at least 2 years. The NBHDs were able to perform a static apnea longer than 3 minutes, a dynamic apnea longer than 70 meters, and practiced apnea for at least 6 months and less than 1 year. None of the breath-hold divers showed decompression signs or suffered from any known decompression sickness during their career.

***Insert Table 1 about here***

\section{Materials and procedures}

A session of both computerized and pencils tests was proposed to the participants, focusing on mnemonic, executive functions and attentional processes. The tests were administered according to publisher's instructions, and were conducted in a quiet room in the psychology department. Tasks evaluating episodic memory functions were WMS-3 Word list learning, recall and recognition (Wechsler 1997) and Rey figure learning and recall (Rey 1964). Working memory was assessed by WMS-3 Letter / number sequencing. Executive functions (inhibition capacity) were assessed by the Stroop modified test (MacLeod 1991). Three computerized tasks were added and programmed with E-prime 2 (Psychology Software Tools, Inc. Pittsburgh, US). Attention and speed processing were assessed via simple visual reaction time, information processing and response inhibition were assessed via a binary choice task, and a mathematical processing was used to measure attention and deductive reasoning.

\section{Written tests}

Twelve words were read and the subject was asked to recall immediately the list on 4 occasions for the WMS-3 Word list test. Then, a late recall and recognition task was also proposed. The score on all tasks was recorded.

The subject was asked to reproduce a complex figure to the best of their ability for the Rey figure test. Score and numbers of missing components were recorded. 
WMS-3 Letter / number sequencing test: A mixed list of numbers and letters was presented and the subject was asked to repeat the list by saying the numbers first in ascending order and the letters in alphabetical order. The maximal span and the score were recorded.

Modified Stroop test: three cards with 100 stimuli each were presented. In card 1, rectangles of a given color were presented and the subject was asked to name the colour as quickly as possible. In card 2, colors names appeared in black and the subject was asked to read the words as quickly as possible. In card 3 (interference card), colors names appeared in a different ink than the color written with the word, and the subject was asked to name the colour independently of the word written as quickly as possible. The recorded parameters were the time to complete the card using a chronometer as well the number of errors for the 3 cards. An interference score was also calculated (time taken to complete card 3 - time taken to complete card 1).

\section{Computerized Tests}

Visual Reaction Time test: participants were asked to react as quickly as possible whenever a visual stimulus appeared. Stimulus exposure endured until a response (space bar) was given. The inter stimulus interval varied pseudo-randomly from 1 to $7 \mathrm{~s}$ and reaction times were recorded on 20 stimuli on the dominant hand.

Binary choice task: The subject was asked to react as quickly as possible when the cross appeared and not to react when the circle appeared. 32 stimuli were presented (20 targets and 12 distractors) for the dominant hand. The inter stimulus interval varied pseudo-randomly from 1 to $7 \mathrm{~s}$. Reaction time and number of errors (commissions and omissions) were recorded.

Mathematical task: This task was inspired from the Automated Neuropsychological Assessment Metrics test system. All problems required an addition and subtraction sequence in the form of " $\mathrm{x}+$ $\mathrm{y}-\mathrm{z}=$ ". The participant was asked to indicate as fast as possible if the solution to the problem is greater than or less than 5. Only the digits 1 through 9 are used; the correct answer may be any number from 1 to 9 except 5; test problems whose answers are greater than or less than 5 are 
equally probable; cumulative intermediate totals have a positive value; working left to right the same digit cannot appear twice in the same problem. Reaction time and errors were recorded on sixteen problems with an inter stimulus interval of $4 \mathrm{~s}$.

\section{Statistical analysis}

The samples were first tested for normality of distribution and homogeneity of variance with Shapiro-Wilk's test and Levene's test, respectively. Then, Univariate General Linear model was computed to compare results between groups (i.e., EBHD, NBHD and CTRL). Post hoc comparisons adjusted with the Student-Newman-Keuls (SNK) correction were conducted whenever the main effect was significant. Pearson correlations were performed to assess the relationships between static apnea best performance, the number of years of apnea training with the modified Stroop test. A p-value $<0.05$ was considered statistically significant for all analyses. Data are presented as the mean value \pm SD. Statistical analysis was performed by means of Statistica software version 7 (StatSoft, Inc. Tulsa, US).

\section{Results}

Anthropometric measurements, breath-hold diving experience and number of neurological events Anthropometric measurements, breath-holding diving experience and the number of neurological events are presented in table 1. No significant difference was found between the groups for age $(\mathrm{F}(2,33)=0.604, \mathrm{MSE}=30.40, \mathrm{p}=0.55)$, education $(\mathrm{F}(2,33)=0.489, \mathrm{MSE}=2.783, \mathrm{p}=0.61)$, height $(\mathrm{F}(2,33)=0.38, \mathrm{MSE}=48, \mathrm{p}=0.68)$ and weight measures $(\mathrm{F}(2,33)=2090.199, \mathrm{MSE}=98.2$, $\mathrm{p}=0.31$ ). Besides, unpaired t-test revealed that the total number of neurological events was higher in EBHD than in NBHD $(\mathrm{p}<0.01)$.

\section{Cognitive results}

Cognitive results are displayed in Table 2. Based on published norms (Wechsler 1997 and Rey 
1964), no pathological scores was found in WMS-3 Word list learning, recall and recognition, Rey figure learning and recall and WMS-3 Letter / number sequencing. However, 1 EBHD presented a pathological score on the modified Stroop test with the greatest number of errors.

There was no significant difference between the 3 groups for the WMS-3 Word list learning, recall and recognition, Rey figure learning and recall and WMS-3 Letter / number sequencing tests. However, General Linear Model revealed a significant main effect in the time to complete the interference card test $(\mathrm{F}(2,32)=3.746, \mathrm{MSE}=201.451, \mathrm{p}<0.05)$. Post hoc analysis revealed that EBHD were slower to perform the interference card test than the two other groups (all $\mathrm{p}<0.05$ ). In addition, a significant main effect was found in the score of the modified stroop test $(F(2,32)=$ 3.931, MSE $=181.343, \mathrm{p}<0.05)$. More precisely, the interference score performed by EBHD was lower than the ones performed by the two other groups (all $\mathrm{p}<0.05$ ).

***Insert Table 2 about here***

Finally, Pearson's analysis showed that the time taken to complete the interference card test was positively correlated with the best performance in maximal static apnea $(r=0.73, p<0.05$, Figure 1) and the number of years of breath-hold diving training $(r=0.79, p<0.001$, Figure 2). However, no linear correlation was found between personal best performance in constant weight and the time taken to complete the interference card test $(\mathrm{p}>0.05)$.

$* * *$ Insert figure 1 about here***

\section{Discussion}

This study investigated the impact of long-term training in free diving on neurocognitive functions from 3 different groups of men with distinct apnea performances. The main findings were that truly elite $\mathrm{BH}$ divers, capable of holding an average breath of $371 \mathrm{sec}$ (128 sec longer than novice divers), were slower to perform the interference card of a Stroop test, and presented more errors and 
a lower total interference score than others participants. Correlation data further suggest that the longer the $\mathrm{BH}$ diving career and static apnea duration, the poorer the score on the Stroop test. Interestingly, the diver who presented a pathological score also had the longest diving career with 19 years and the best static apnea with $436 \mathrm{sec}$. There was no difference between novice BH divers and the control group on any neurological tests. These findings indicate mild short-term memory impairments likely related to the years of diving training.

A poorer score and longer time to perform the interference card of a Stroop test indicates a lesser inhibitory capacity of automatic processes (Diamond and Ling 2016). This is the first time that elite BH divers are demonstrated to perform more poorly on this test (including a pathological score), compared with novice $\mathrm{BH}$ divers and controls. The prefrontal cortex and caudate and sub-thalamic nuclei are involved in inhibitory control cognition (Swick and Jovanovic 2002, Diamond 2013). The brain requires a constant supply of $\mathrm{O}_{2}$ to meet metabolic demands that is maintained through a series of efficient cerebral homeostatic and vascular mechanisms. Hypoxia has several debilitating effects on brain functions that span from mild acute alterations to severe chronic impairments (Caine and Watson 2000, Hornbein 2001). Of particular interest to the present results is that there may be a characteristic memory deficit associated with lesions of the prefrontal cortex and caudate nucleus, characterized by poor recall with relative preservation of recognition abilities (Cummings 1993). Following a mountain ascent, a decline in visual and verbal long-term memory, as well as cortical atrophy have been reported (Hornbein et al. 1989; Fayed et al. 2006). Along the same line, aviation pilots exhibited decreased perceptual ability under hypoxic conditions corresponding to $5000 \mathrm{~m}$ altitude that worsened with progressively lower arterial $\mathrm{O}_{2}$ saturation (Truszczyński et al. 2009). Closer to the sport of free diving, when triathletes were asked to estimate/recognise specific durations while holding their breath, they consistantly overestimated time (Jamin et al. 2004).

There is also strong evidence that even a transient hypoxia subsequent to maximal static $\mathrm{BH}$ 
performance may lead to mild brain damages. For example, a significant increase in [S100B] protein (a serum marker for cerebral ischemia and brain damage) was demonstrated after a voluntary prolonged apnea (mean apneic time: $335 \mathrm{sec}$, range 281-403 sec) in trained $\mathrm{BH}$ divers (Andersson et al. 2009). The authors concluded that the increase in S100B was caused by asphyxia and/or the increase in blood pressure. Notably, [S100B] is still significantly elevated five days after a dynamic apnea that resulted in hypoxic syncope (Linér and Andersson 2009), highlighting the long-lasting negative consequences of a NNE. Such findings were confirmed by Gren and colleagues (Gren et al. 2016) who reported an altered amyloid metabolism reflecting neuronal damage or dysfunction after a maximal static apnea (mean apneic time: $302 \mathrm{sec}$, range 138-457 sec) performed during a national championship. An accumulation of amyloid precursor protein is typically found in patients with traumatic brain injury (Baugh et al. 2012), which strengthens the evidence for the strong debilitating impact of a BH episode held to one's maximal capacity for the integrity of the central nervous system. Interestingly, the level of total tau structural protein, which serves to maintain grey matter cortical interneurons integrity and functionality, was also positively correlated with apneic time (Gren et al. 2016). One may therefore argue that repeated exposures to such transient, severe hypoxia leads to chronic negative consequences in diver populations. In fact, a brain imaging study reinforces that hypothesis by showing atypical tomographic scans, indicating brain functions abnormalities in 5 elite BH divers (Potkin and Uszler 2006). The current results of altered short-term memory are therefore in good agreement with such findings, suggesting that acute apnea may negatively impact the cognitive function over several years.

By contrast to high-altitude environment (i.e. poïkilocapnic hypoxia), prolonged apnea is associated with progressive increase in arterial carbon dioxide partial pressure that might ultimately alter dynamic cerebral autoregulation in EBHDs (Cross et al. 2014). As a result, repeated episodes of severe hypercapnia might also contribute to brain damage over the years in EBHDs, resulting in fine in mild neuropsychological sequelae.

While we report some specific cognitive alterations in elite, but not in novice divers and controls, 
another study rather concluded to the absence of neuropsychological sequalae in divers (Ridgway and McFarland 2006). However, the authors emphasised that further studies should be conducted before concluding that long-duration apneas are without consequences. While these findings may appear conflicting, it is possible that the training level of the investigated divers played a confounding role. In fact, our participants could perform maximal apneas of more than 6 min on average (with maximum of $7 \mathrm{~min} 33 \mathrm{sec}$ ), which is well above the times measured in the above studies reporting physiological alterations of the central nervous system. It is therefore likely that this group of elite divers constantly faces severe hypoxia during training, which would certainly augment their likelihood of developing neurocognitive impairments over the course of their career. In fact, the current data highlights the static apneic time, but not the number of negative neurological events, as a strong predictor of cognitive impairment. Overall, this shows that the number of diving training years and neurological events might not be sufficient or valid criterion when studying elite divers, and that performance criterion (e.g., static apneic time $>360 \mathrm{sec}$ ) should be taken into account to distinguish between divers with vs. without potential for neuropsychological impairments.

\section{Conclusion}

This study demonstrates for the first time poorer scores on the Stroop test in elite BH divers than in novice divers and non-diving controls. The present scores were also correlated with the best static apneic time, indicating that the best divers displayed the greatest alteration of the short-term memory. These findings suggest the possibility that elite breath-hold divers practising apnea over several years may display mild short-term memory impairments.

\section{Conflict of interest disclaimer}

The authors report no conflicts of interest associated with this manuscript. 


\section{Acknowledgments}

We acknowledge with gratitude the contribution of all the participants: recordmen, beginners or anonymous.

\section{References}

Andersson, J.P., Liner, M.H., and Jonsson, H. 2009. Increased serum levels of the brain damage marker S100B after apnea in trained breath-hold divers: a study including respiratory and cardiovascular observations. J. Appl. Physiol. 107(3): 809-815. doi:10.1152/japplphysiol.91434.2008.

Auer, R.N., and Sutherland, G.R. 2002. Hypoxia and related conditions. In Greenfields neuropathology, 7 edition. Edited by D.I. Graham and P.L. Lantos. pp. 233-280.

Baugh, C.M., Stamm, J.M., Riley, D.O., Gavett, B.E., Shenton, M.E., Lin, A., Nowinski, C.J., Cantu, R.C., McKee, A.C., and Stern, R.A. 2012. Chronic traumatic encephalopathy: neurodegeneration following repetitive concussive and subconcussive brain trauma. Brain Imag. Behav. 6(2): 244-254. doi:10.1007/s11682-012-9164-5.

Caine, D., and Watson, J. 2000. Neuropsychological and neuropathological sequelae of cerebral anoxia: A critical review. J. Int. Neuropsychol. Soc. 6: 86-89.

Cross TJ, Kavanagh JJ, Breskovic T, Johnson BD, Dujic Z (2014) Dynamic Cerebral Autoregulation Is Acutely Impaired during Maximal Apnoea in Trained Divers. PloS one, 9 (2):e87598. doi:10.1371/journal.pone.0087598

Cummings, J.L. 1993. Frontal-subcortical circuits and human behavior. Arch. Neurol. 50: 873-880.

Diamond, A. 2013. Executive Functions. Annu. Rev. Psychol. 64(1): 135-168. doi:10.1146/annurev-psych-113011-143750.

Diamond, A., and Ling, D.S. 2016. Conclusions about interventions, programs, and approaches for improving executive functions that appear justified and those that, despite much hype, do not. Dev. Cogn. Neurosci. 18: 34-48. doi:10.1016/j.den.2015.11.005.

Fayed, N., Modrego, P.J., and Morales, H. 2006. Evidence of Brain Damage after High-altitude Climbing by Means of Magnetic Resonance Imaging. Am. J. Med. 119(2): 168.e1-168.e6. Elsevier. doi:10.1016/j.amjmed.2005.07.062.

Gren, M., Shahim, P., Lautner, R., Wilson, D.H., Andreasson, U., Norgren, N., Blennow, K., and Zetterberg, H. 2016. Blood biomarkers indicate mild neuroaxonal injury and increased amyloid Bproduction after transient hypoxia during breath-hold diving. Brain Inj. 30(10): 1226-1230. doi:10.1080/02699052.2016.1179792.

Hornbein, T.F. 2001. The high-altitude brain. J. Exp. Biol. 204(Pt 18): 3129-3132.

Hornbein, T.F., Townes, B.D., Schoene, R.B., Sutton, J.R., and Houston, C.S. 1989. The Cost to the Central Nervous System of Climbing to Extremely High Altitude. New Engl. J. Med. 321(25): 1714-1719.

Jamin, T., Joulia, F., Fontanari, P., Giacomoni, M., Bonnon, M., Vidal, F., and Crémieux, J. 2004. Apnea-induced changes in time estimation and its relation to bradycardia. Aviat. Space Environ. Med. 75(10): 876-880.

Lindholm, P. 2007. Loss of Motor Control and/or Loss of Consciousness during Breath-Hold Competitions. Int. J. Sports Med. 28(4): 295-299. doi:10.1055/s-2006-924361.

Linér, M.H., and Andersson, J.P.A. 2009. Hypoxic Syncope in a Competitive Breath-Hold Diver with Elevation of the Brain Damage Marker S100B. Aviat. Space Environ. Med. 80(12): 10661068. doi:10.3357/ASEM.2554.2009.

MacLeod, C.M. 1991. Half a century of research on the Stroop effect: an integrative review. Psychological Bulletin, 109(2): 163-203. 
Potkin, R.T., and Uszler, J.M. 2006. Brain function imaging in asymptomatic elite breath-hold divers. In Breath-Hold Diving. Proceedings of the Undersea and Hyperbaric Medicine, Edited by P. Lindholm, N.W. Pollock, and C. Lundgren. Durham, NC. pp. 135-136.

Rey, A. 1964. L'examen clinique en psychologie. In 2nd edition. Presses universitaires de France, Paris.

Ridgway, L., and McFarland, K. 2006. Apnea Diving: Long-Term Neurocognitive Sequelae of Repeated Hypoxemia. Clin. Neuropsychol. 20(1): 160-176. doi:10.1080/13854040590947407.

Swick, D., and Jovanovic, J. 2002. Anterior cingulate cortex and the Stroop task: neuropsychological evidence for topographic specificity. Neuropsychologia, 40: 1240-1253.

Truszczyński, O., Wojtkowiak, M., Biernacki, M., and Kowalczuk, K. 2009. The effect of hypoxia on the critical flicker fusion threshold in pilots. Int. J. Occup. Med. Environ. Health 22(1): $13-$ 18. doi:10.2478/v10001-009-0002-y.

Wechsler, D. 1997. Wechsler Memory Scale-Third Edition. The Psychological Corporation, San Antonio, TX. 


\section{Tables}

Table 1. Demographic, anthropometric and apnea diving experience for the three groups $(n=36)$

\begin{tabular}{|c|c|c|c|c|c|c|c|c|c|}
\hline & \multicolumn{3}{|c|}{$\begin{array}{l}\text { Elite Breath-Hold Divers } \\
\qquad(\mathrm{n}=12)\end{array}$} & \multicolumn{3}{|c|}{$\begin{array}{l}\text { Novice Breath-Hold } \\
\text { Divers }(n=12)\end{array}$} & \multicolumn{3}{|c|}{$\begin{array}{l}\text { Non-diving Controls } \\
\qquad(\mathrm{n}=12)\end{array}$} \\
\hline & Range & Mean & SD & Range & Mean & SD & Range & Mean & SD \\
\hline Age (years) & $24-49$ & 32.41 & 6.43 & $23-41$ & 30 & 4.84 & $24-41$ & 30.75 & 5.13 \\
\hline Education (years) & $12-17$ & 15.08 & 1.83 & $12-17$ & 15.33 & 1.66 & $12-17$ & 15.75 & 1.48 \\
\hline Height (cm) & $172-193$ & 179 & 5.73 & $167-190$ & 178,16 & 6.96 & $170-195$ & 180.58 & 7.93 \\
\hline Body mass (kg) & $60-95$ & 74.58 & 10.50 & $56-84$ & 72.91 & 8.03 & $62-96$ & 79 & 10.93 \\
\hline Months engaged in apnea & $24-228$ & 105 & 64.13 & $4-12$ & 8.75 & 2.63 & -- & -- & -- \\
\hline SA best performance $(\mathrm{sec})$ & $300-439$ & 371 & 43.83 & $180-295$ & 243 & 34.50 & -- & -- & -- \\
\hline DA best performance (m) & $95-223$ & 137.75 & 40.46 & $65-76$ & 71.75 & 3.36 & -- & -- & -- \\
\hline CWT best performance (m) & $62-98$ & 79.91 & 11.41 & $15-29$ & 20.33 & 4.08 & -- & -- & -- \\
\hline Apnea training (hours/week) & $1-10$ & 3.16 & 2.48 & $2-4$ & 2.58 & 0.66 & -- & -- & -- \\
\hline Total number of LOC & $0-5$ & 1.16 & 1.40 & 0 & 0 & -- & -- & -- & -- \\
\hline Total number of LMC & $0-10$ & 2.75 & 2.73 & $0-2$ & 0.25 & 0.62 & -- & -- & -- \\
\hline Total number of NNE & $0-15$ & 3.91 & 3.91 & $0-2$ & 0.25 & 0.62 & -- & -- & -- \\
\hline
\end{tabular}

SA: static apnea; DA: dynamic apnea; CWT: constant weight; LOC: loss of consciousness; LMC: loss of motor control; NNE: negative neurological events (include LOC and LMC). 


\section{Table 2}

Means (standard deviations). Mean Square Error and F-Values on neuropsychological tests for the three groups ( $\mathrm{n}=36$ )

\begin{tabular}{|c|c|c|c|c|c|c|}
\hline Neuropsychological test variable & Elite Breath-Hold Divers & Novice Breath-Hold Divers & Non Breath-Hold Divers & $\mathrm{F}(2.33)$ & MSE & $\mathrm{p}$-value \\
\hline \multicolumn{7}{|l|}{ WMS-3 Word list } \\
\hline Maximal score (Trial 1-4) & $10.66(1.30)$ & $10.66(1.07)$ & $11(1.12)$ & 0.324 & 1.374 & .72 \\
\hline Score on trial 1 & $6.58(1.56)$ & $6.83(1.46)$ & $7.16(1.46)$ & 0.456 & 2.250 & .63 \\
\hline Total immediate score & $36.16(3.88)$ & $37(3.21)$ & $37.58(4.69)$ & 0.384 & 15.840 & .68 \\
\hline Late recall Score & $8.41(1.97)$ & $9.08(1.83)$ & $9.25(2.49)$ & 0.520 & 4.480 & .59 \\
\hline Recognition Score & $23.50(0.67)$ & $23.83(0.38)$ & $23.33(0.88)$ & 1.670 & 0.460 & .2 \\
\hline \multicolumn{7}{|l|}{ Rey Figure } \\
\hline Recall Score & $28.41(3.28)$ & $27.83(4.28)$ & $30.75(4.35)$ & 1.784 & 16.030 & .18 \\
\hline Number of errors & $2.75(1.42)$ & $2.33(1.49)$ & $1.75(1.65)$ & 1.290 & 2.330 & .28 \\
\hline Number of missing components & $2.41(1.31)$ & $2.91(2.02)$ & $1.75(1.54)$ & 1.500 & 2.720 & .23 \\
\hline \multicolumn{7}{|l|}{ WMS-3 Letter/number sequencing } \\
\hline Score & $12.50(2.86)$ & $11.33(1.82)$ & $12.58(1.78)$ & 1.175 & 4.980 & .32 \\
\hline Maximal span & $5.91(0.90)$ & $5.41(0.66)$ & $6(0.85)$ & 1.805 & 0.662 & .18 \\
\hline \multicolumn{7}{|l|}{ Stroop-Modified } \\
\hline Time card 1 & $53.16(7.06)$ & $54.16(7.05)$ & $52.41(5.75)$ & 0.209 & 44.300 & .81 \\
\hline Number of errors card 1 & $0(0)$ & $0(0)$ & $0(0)$ & -- & -- & -- \\
\hline Time card 2 & $41.66(6.67)$ & $41.83(5.82)$ & $41.50(5.45)$ & 0.009 & 36.220 & .99 \\
\hline Number of errors & $0(0)$ & $0(0)$ & $0(0)$ & -- & -- & -- \\
\hline Time Card 3 & $101.73(14.0)$ & $87(12.64)$ & $88.25(15.76)$ & 3.746 & 201.451 & .035 \\
\hline Number of errors & $0.58(1.24)$ & $0.08(0.2)$ & $0(0)$ & 2.210 & 0.540 & .12 \\
\hline Score Interference & $47.82(14.70)$ & $32.83(9.05)$ & $35.83(15.78)$ & 3.931 & 181.343 & .03 \\
\hline Visual reaction time & $267(41.81)$ & $278(23.17)$ & $300(41)$ & 2.429 & 1329.000 & .1 \\
\hline \multicolumn{7}{|l|}{ Binary choice task } \\
\hline Reaction Time & $437(40.80)$ & $448(58.83)$ & $452(69.42)$ & 0.208 & 3316.000 & .81 \\
\hline
\end{tabular}




\section{Applied Physiology, Nutrition, and Metabolism}

Number of errors

$0.75(1.05)$

$0.75(0.75)$

$0.83(1.11)$

0.020

0.970

0.97

Mathematical task

Reaction Time

2390 (508.75)

2195 (487.18)

$2185(583)$

0.570

1.83 (194)

0.620

278694.000

2.440

.56

$2.08(1.37)$

https://mc06.manuscriptcentral.com/apnm-pubs 


\section{Figure captions}

Figure 1. Linear relationship between the best performance in maximal static apnea (SA) and the time taken to complete the interference card of the Stroop test in elite breath-hold divers $(n=12)$. The diver displayed with a plain circle had the longest SA duration (436 sec) and presented a pathological score on the Stroop test.

Figure 2. Linear relationship between the number of years of breath-hold diving practice (YAP) and the time taken to complete the interference card test on the Stroop test in elite breath-hold divers $(n=12)$. The diver displayed with a plain circle had the longest SA duration (436 sec), the longest diving career (19 years), and presented a pathological score on the Stroop test. 


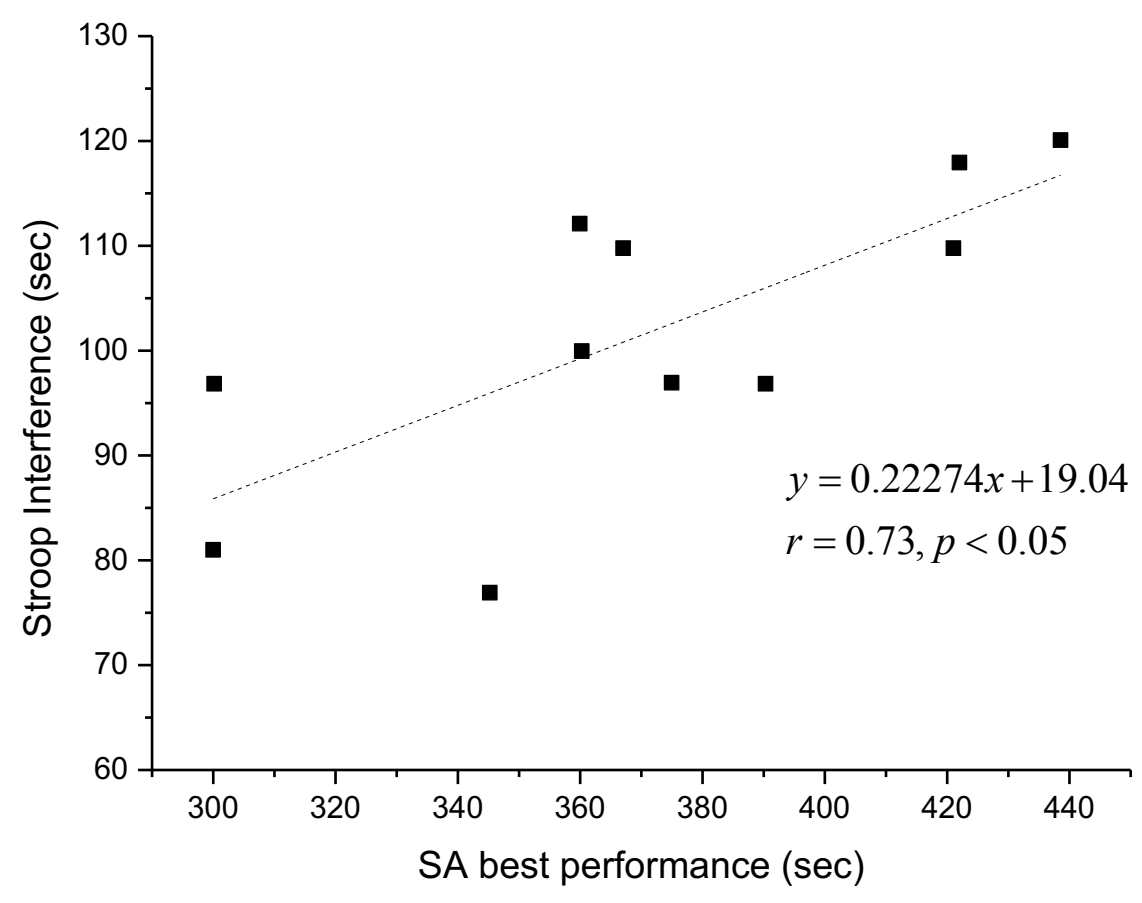




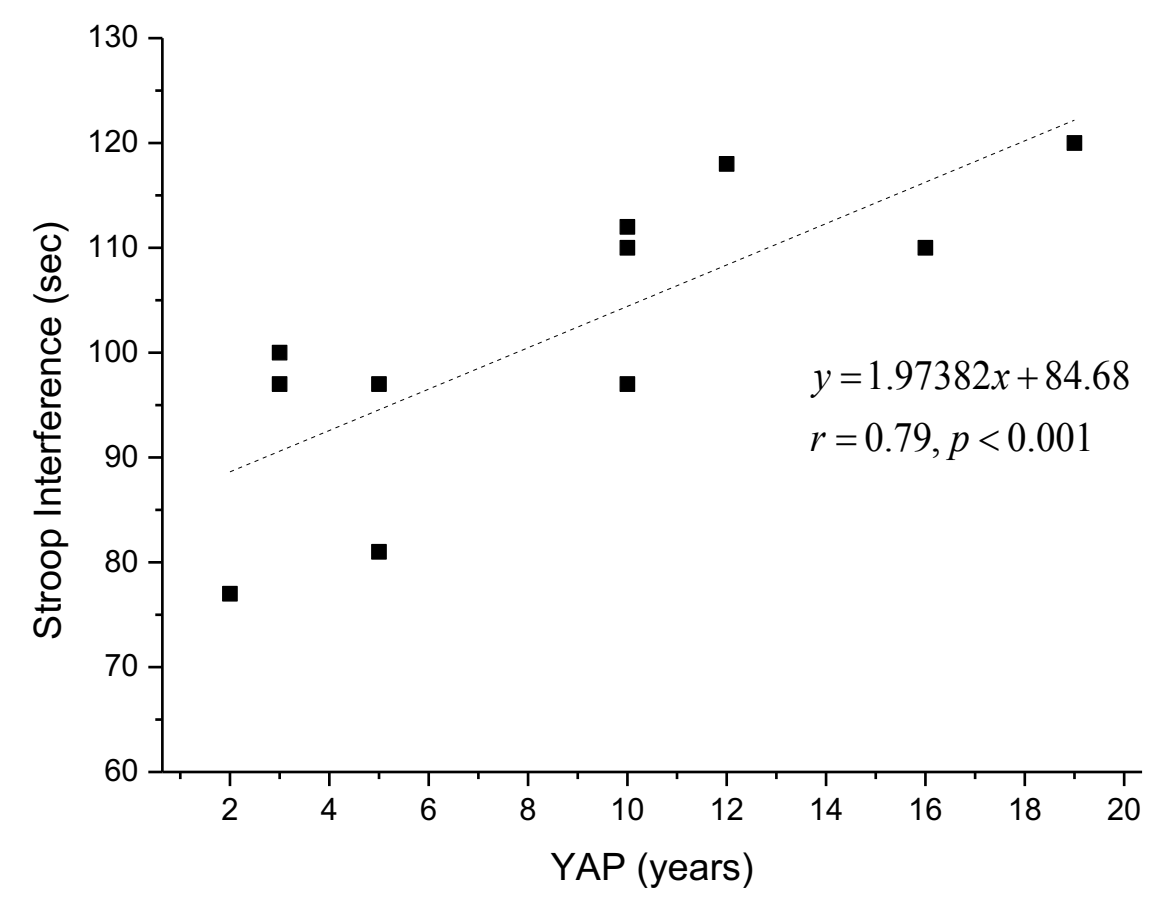

